\title{
Uso transitorio de sistemas personalizados de dosificación (SPD): más allá de la polimedicación
}

\author{
Inés Mera Gallego', Eduardo J. Satué de Velasco \\ 1. Farmacéutica comunitaria. Farmacia Satué, Maella (Zaragoza). Graduada en Nutrición Humana y Dietética. Grupo Berbés de Investigación y \\ Docencia en Atención Farmacéutica. Grupos de Diabetes y Nutrición y Digestivo de SEFAC. 2. Farmacéutico comunitario. Farmacia Satué, Maella \\ (Zaragoza).
}

\section{PALABRAS CLAVE}

Polimedicación, cambios en el tratamiento, sistema personalizado de dosificación, farmacéutico comunitario, servicios profesionales farmacéuticos

\section{ABREVIATURAS}

AD: administración a demanda

E: efectividad

MAP: médico de atención primaria

$\mathrm{N}$ : necesidad

PA: principio activo

PRM: problema relacionado con la medicación

RAM: reacción adversa a medicamentos

RNM: resultado negativo de la medicación S: seguridad

SPD: sistema personalizado de dosificación SPF: servicios profesionales

farmacéuticos

\section{RESUMEN}

Los pacientes diana del Servicio Personalizado de Dosificación (SPD) han sido habitualmente las personas con enfermedades crónicas polimedicadas con dificultades físicas o cognitivas para manejar adecuadamente su medicación. Sin embargo, existen otros grupos susceptible de beneficiarse igualmente de esta herramienta, como son los pacientes sin deficiencias ni dependencias pero que debido a la complejidad de la pauta del tratamiento a administrar pueden aprovechar este servicio de forma puntual. En este caso veremos una paciente habituada a manejar no sólo su medicación sino también la de su marido pero que debido a una pauta cambiante de medicamentos ansiolíticos y antidepresivos prefiere confiar en la farmacia comunitaria para su correcta administración.

\section{Antecedentes}

Mujer de 64 años, con tratamiento para la hipercolesterolemia primaria, trastorno de depresión y ansiedad, dolor neuropático, hipertensión arterial y prediabetes. La paciente es habitual de la farmacia, participando en diversos servicios profesionales farmacéuticos (SPF) como consulta farmacéutica o en el servicio de control de la hipertensión arterial, realizando mediciones aisladas en la farmacia y monitorización ambulatoria. Presenta además colon irritable que suele generarle iatrogenia con los tratamientos introducidos (y determinados alimentos) por lo que es frecuente la consulta farmacéutica por reacciones adversas a medicamentos (RAM). Alergias diagnosticadas: plátano.

\section{Razón de consulta}

La paciente sufre diarreas desde hace un mes, por lo que consultó en la farmacia el motivo y tras realizar una revisión de la medicación, se consideró la posibilidad de que paroxetina, introducida unos tres meses antes para el tratamiento de la depresión, fuese la causa, tal como ya le había ocurrido en otras ocasiones con otros tratamientos. La paciente concierta una visita a su psiquiatra que decide eliminarle paulatinamente paroxetina e ir introduciendo sertralina. Además, debido al ajuste serotoninérgico, también le plantea un reajuste de la pauta ansiolítica. De vuelta a la farmacia, la paciente está muy afectada, puesto que las malas experiencias previas le generan desconfianza en su capacidad para tolerar nuevos medication, personalized dosage pharmaceutical services 
cambios y reconoce la dificultad para gestionarlos adecuadamente, pese a que habitualmente ella misma se realiza su "pastillero" semanal y el de su marido. Por estas razones se le ofrece la inclusión en el servicio de mejora de la adherencia mediante el uso del sistema personalizado de dosificación (SPD). El objetivo del ofrecimiento es sobre todo tranquilizar y ayudar a superar el momento de confusión, más que la inclusión a largo plazo en el SPF, dado que es una persona válida. El SPD se elabora siguiendo el Procedimiento Normalizado de Trabajo de Sistemas Personalizados de Dosificación del Colegio Oficial de Farmacéuticos de Zaragoza y en cumplimiento del decreto aragonés 93/2015 [1], que incluye, además de la operativa del SPD, una revisión de la farmacoterapia.

\section{Análisis, evaluación e intervenciones}

\section{Situación inicial (11/08/17)}

En la entrevista con la paciente se registran los problemas de salud y los medicamentos que utiliza (tabla 1).

\section{Evaluación inicial}

Tras la revisión de las fichas técnicas de los medicamentos pautados [2] se considera posible que la paciente presente un resultado negativo de la medicación (RNM) no cuantitativo, la diarrea, debido a la medicación antidepresiva, paroxetina. Según la ficha técnica de paroxetina, las diarreas como reacción adversa a esta medicación son frecuentes $(>1 / 100$, $<1 / 10$ ) [3]. Por ello, la psiquiatra propone los siguientes cambios:

- Retirada gradual de paroxetina e introducción gradual de sertralina:

- Paroxetina 20 mg: tomar medio comprimido durante una semana y retirar.

- Sertralina $50 \mathrm{mg}$ : al retirar paroxetina $20 \mathrm{mg}$ introducir sertralina $50 \mathrm{mg}$, un comprimido por la mañana durante una semana y aumentar a dos comprimidos la siguiente semana.

- Subida de la dosis de ansiolítico hasta reajuste de la pauta antidepresiva:

- Alprazolam 0,5 mg: aumentar la dosis a dos comprimidos por la mañana, disminuir a un com- primido la siguiente semana $\mathrm{y}$ rebajar hasta $1 / 2$ comprimido la siguiente semana. El resto de las tomas del día continuar igual. Este cambio de pauta es el que genera más inquietud por miedo a no hacerlo correctamente e influir negativamente en el tratamiento de la ansiedad.

Tiene un problema de salud no tratado por necesidad de tratamiento de la diarrea.

No existe ninguna duplicidad, ni interacción entre los fármacos prescritos.

\section{Intervención inicial}

Se procede a tratar el problema de la diarrea con recomendaciones de dieta astringente, correcta hidratación, suero y con el uso de probióticos debido a sus diarreas recurrentes.

Se realiza el SPD teniendo en cuenta que mianserina $30 \mathrm{mg}$ es un fármaco no emblistable [2], algo que la paciente desconocía (por lo que los desemblistaba en su "pastillero" casero generando

Tabla 1 Problemas de salud y medicamentos que utiliza la paciente

\begin{tabular}{|c|c|c|c|c|c|c|c|c|}
\hline \multicolumn{3}{|c|}{ Problemas de salud } & \multicolumn{6}{|c|}{ Medicamentos } \\
\hline Inicio & Problemas de salud & Controlado & Inicio & Medicamento (PA) & Pauta & $\mathrm{N}$ & $\mathrm{E}$ & S \\
\hline 2015 & Hipercolesterolemia primaria & sí & $01 / 15$ & Ezetimiba $10 \mathrm{mg}$ & $0-0-1-0$ & Si & Si & Si \\
\hline \multirow{3}{*}{2016} & \multirow{3}{*}{$\begin{array}{l}\text { Trastorno depresivo asociado } \\
\text { a ansiedad }\end{array}$} & \multirow{3}{*}{ ¿? } & $04 / 17$ & Mianserina $30 \mathrm{mg}$ & $0-0-0-1$ & Si & ¿? & Si \\
\hline & & & $05 / 17$ & Paroxetina $20 \mathrm{mg}$ & $1-0-0-0$ & Si & ¿? & No \\
\hline & & & $02 / 16$ & Alprazolam 0,5 mg & $1-1 / 2-0-1$ & Si & ¿? & Si \\
\hline 2017 & Dolor neuropático periférico & Si & $03 / 17$ & Gabapentina $300 \mathrm{mg}$ & $1-0-0-1$ & Si & Sí & Si \\
\hline \multirow{2}{*}{2012} & \multirow{2}{*}{ Hipertensión arterial } & \multirow{2}{*}{ Si } & $05 / 12$ & Lisinopril 5 mg & $1-0-1-0$ & Si & Si & Si \\
\hline & & & $02 / 16$ & Nevibolol 5 mg & $0-1-0-1$ & Si & Sí & Si \\
\hline 2011 & Insomnio & Si & 2012 & Lorazepam $1 \mathrm{mg}$ & $A D$ & Si & Si & Sí \\
\hline $07 / 2017$ & Diarrea & No & & & & & & \\
\hline
\end{tabular}

AD: a demanda; E: efectividad; N: necesidad; PA: principio activo; S: seguridad. 
Tabla 2 Ficha del paciente para el primer SPD

\begin{tabular}{|c|c|c|c|c|c|c|c|c|}
\hline Medicamento & Posología & $\begin{array}{c}\text { Modo de } \\
\text { administración }\end{array}$ & $\begin{array}{c}\text { Vía de } \\
\text { administración }\end{array}$ & $\begin{array}{l}\text { Médico } \\
\text { prescriptor }\end{array}$ & $\begin{array}{c}\text { Fecha de inicio } \\
\text { tratamiento }\end{array}$ & $\begin{array}{l}\text { Fecha prevista } \\
\text { finalización }\end{array}$ & Incidencias & $\begin{array}{l}\text { Blister } \\
\text { Si/No }\end{array}$ \\
\hline $\begin{array}{l}\text { Ezetimiba } \\
10 \mathrm{mg}\end{array}$ & $0-0-1-0$ & $\begin{array}{l}\text { Con o sin } \\
\text { alimentos }\end{array}$ & Oral & MAP & $01 / 15$ & & & Si \\
\hline $\begin{array}{l}\text { Miansenina } \\
30 \mathrm{mg}\end{array}$ & $0-0-0-1$ & $\begin{array}{l}\text { Con o sin } \\
\text { alimentos }\end{array}$ & Oral & Psiquiatra & $04 / 17$ & & & No \\
\hline $\begin{array}{l}\text { Paroxetina } \\
20 \mathrm{mg}\end{array}$ & $1 / 2-0-0-0$ & $\begin{array}{l}\text { Con o sin } \\
\text { alimentos }\end{array}$ & Oral & Psiquiatra & $05 / 17$ & 20/08/17 & & Sí \\
\hline $\begin{array}{l}\text { Alprazolam } \\
0,5 \mathrm{mg}\end{array}$ & $2-1 / 2-0-1$ & $\begin{array}{l}\text { Con o sin } \\
\text { alimentos }\end{array}$ & Oral & Psiquiatra & $02 / 16$ & & & Sí \\
\hline $\begin{array}{l}\text { Gabapentina } \\
300 \text { mg }\end{array}$ & $1-0-0-1$ & $\begin{array}{l}\text { Con o sin } \\
\text { alimentos }\end{array}$ & Oral & MAP & $03 / 17$ & & & Si \\
\hline $\begin{array}{l}\text { Lisinopril } \\
5 \mathrm{mg}\end{array}$ & $1-0-1-0$ & $\begin{array}{l}\text { Con o sin } \\
\text { alimentos }\end{array}$ & Oral & MAP & $05 / 12$ & & & Si \\
\hline $\begin{array}{l}\text { Nebivolol } \\
5 \mathrm{mg}\end{array}$ & $0-1-0-0$ & $\begin{array}{l}\text { Con o sin } \\
\text { alimentos }\end{array}$ & Oral & MAP & $02 / 16$ & & & Sí \\
\hline $\begin{array}{l}\text { Lorazepam } \\
1 \mathrm{mg}\end{array}$ & A demanda & $\begin{array}{l}\text { Con o sin } \\
\text { alimentos }\end{array}$ & Oral & MAP & 2012 & & & No \\
\hline
\end{tabular}

MAP: Médico de Atención Primaria.

un problema relacionado con la medicación (PRM de mala conservación).

Se propone un programa de SPD de cuatro semanas hasta la finalización de los reajustes indicados por la psiquiatra. La ficha del paciente para el primer SPD se muestra en la tabla 2.

A su vez, se realiza una revisión de la farmacoterapia, sin detectar nada más reseñable.
Situación tras una semana con SPD Las variaciones del tratamiento habitual que fueron introducidas en el primer SPD se muestran en la tabla 3.

\section{Evaluación tras una semana} con SPD

La paciente acude a la farmacia comunitaria para recoger su segun- do SPD. Se le pregunta por el tratamiento de la diarrea y nos comenta que ha cesado, consiguiendo normalidad en el proceso de evacuación.

No tiene ningún problema a la hora de conseguir la adherencia al tratamiento con el SPD. No tiene problemas a la hora de acordarse de tomar mianserina $30 \mathrm{mg}$ fuera del SPD.

Tabla 3 Cambios en el tratamiento introducidos en el primer SPD

\begin{tabular}{|c|l|c|c|c|c|c|c|c|}
\hline \multicolumn{3}{|c|}{ Problemas de salud } & \multicolumn{5}{c|}{ Medicamentos } \\
\hline Inicio & Problemas de salud & Controlado & Inicio & Medicamento (PA) & $\begin{array}{c}\text { Pauta } \\
\text { prescr. }\end{array}$ & N & E & S \\
\hline 2016 & Trastorno depresivo (ansiedad) & i? & $05 / 17$ & Paroxetina $20 \mathrm{mg}$ & $1 / 2-0-0-0$ & Si & i? & No \\
\hline & & & $04 / 17$ & Mianserina $30 \mathrm{mg}$ & $0-0-0-1$ & Si & i? \\
\hline $06 / 17$ & Diarrea & & $02 / 16$ & Alprazolam $0,5 \mathrm{mg}$ & $2-1 / 2-0-1$ & Si & i? & Si \\
\hline
\end{tabular}


Tabla 4 Cambios en el tratamiento introducidos en el segundo SPD

\begin{tabular}{|c|c|c|c|c|c|c|c|c|}
\hline \multicolumn{3}{|c|}{ Problemas de salud } & \multicolumn{5}{c|}{ Medicamentos } \\
\hline Inicio & Problemas de salud & Controlado & Inicio & Medicamento (PA) & $\begin{array}{c}\text { Pauta } \\
\text { prescr. }\end{array}$ & N & E \\
\hline 2016 & Trastorno depresivo (ansiedad) & i? & $21 / 08 / 17$ & Sertralina $50 \mathrm{mg}$ & $1-0-0-0$ & Si & i? & No \\
\hline & & & $04 / 17$ & Mianserina $30 \mathrm{mg}$ & $0-0-0-1$ & Si & i? & Si \\
\hline
\end{tabular}

Intervención tras una semana con SPD

Se produce la eliminación de paroxetina $20 \mathrm{mg}$, se introduce sertralina $50 \mathrm{mg}$ y se mantiene alprazolam $0,5 \mathrm{mg}$

Situación tras dos semanas con SPD

Las variaciones del tratamiento que fueron introducidas en el segundo SPD son: (tabla 4)

\section{Evaluación tras dos semanas} con SPD

La paciente acude a la farmacia comunitaria para recoger su tercer SPD. Se le pregunta si sertralina $50 \mathrm{mg}$ le ha producido algún efecto adverso y si ha vuelto con su estado de diarreas. Nos comenta que todo va bien.

No tiene ningún problema a la hora de cumplir adecuadamente el tratamiento con el SPD, ni problemas a la hora de acordarse de tomar mianserina $30 \mathrm{mg}$.

Intervención tras dos semanas con SPD

Siguiendo las indicaciones de la psiquiatra se introduce un aumento de dosis de sertralina $50 \mathrm{mg}$ y se disminuye alprazolam $0,5 \mathrm{mg}$.
Situación tras tres semanas con SPD

Las variaciones del tratamiento que fueron introducidas en el tercer SPD se muestran a continuación (tabla 5).

\section{Evaluación tras tres semanas} con SPD

La paciente acude a la farmacia comunitaria para recoger su cuarto SPD. Se le pregunta si el aumento de sertralina le ha producido algún efecto adverso y si ha vuelto con su estado de diarreas; nos comenta que han vuelto. Vuelve a tener molestias diarreicas con diverso grado de intensidad.

No tiene ningún problema a la hora de conseguir la adherencia al tratamiento con el SPD, ni problemas a la hora de acordarse de tomar mianserina $30 \mathrm{mg}$.

\section{Intervención tras tres semanas con SPD}

La paciente va mejorando en su estado diarreico, aunque aún sufre episodios puntuales, por lo que sospechamos que paroxetina no era la causante de esta patología, debido a que con sertralina sigue teniendo episodios diarreicos, aunque de menor intensidad.
Informamos a su médico, mediante un informe escrito, de esta evaluación y sugerimos la posibilidad de algún tipo de intolerancia alimentaria. Preparamos el cuarto SPD (tabla 6)

\section{Resultados}

Pasadas las cuatro semanas se alcanza la pauta estable. La paciente aún sigue dos semanas más con el SPF-SPD porque le genera confianza. A partir de entonces la paciente se considera ya capaz de continuar el tratamiento por sus propios medios, lo que le ha permitido mejorar la ansiedad que le provocaba cumplir adecuadamente con la pauta marcada por la psiquiatra, mejorando el control de su trastorno ansioso-depresivo. A su vez, se le recuerda la conveniencia de no desemblistar mianserina hasta su toma.

Tras el informe escrito enviado al médico de atención primaria, en el que se le trasladaba la sospecha de que el problema de diarrea podría tener un origen en una intolerancia alimentaria, este decide realizarle el test correspondiente, resultando positiva una intolerancia al trigo.

Tabla 5 Cambios en el tratamiento introducidos en el tercer SPD

\begin{tabular}{|c|c|c|c|c|c|c|c|c|}
\hline \multicolumn{3}{|c|}{ Problemas de salud } & \multicolumn{6}{|c|}{ Medicamentos } \\
\hline Inicio & Problemas de salud & Controlado & Inicio & Medicamento (PA) & $\begin{array}{l}\text { Pauta } \\
\text { prescr. }\end{array}$ & N & E & $S$ \\
\hline \multirow{3}{*}{2016} & \multirow{3}{*}{ Trastorno depresivo (ansiedad) } & \multirow{3}{*}{ ¿? } & $04 / 17$ & Mianserina 30 mg & $0-0-0-1$ & Si & ¿? & Si \\
\hline & & & $18 / 08 / 17$ & Sertralina $50 \mathrm{mg}$ & $2-0-0-0$ & Si & ¿? & No \\
\hline & & & $02 / 16$ & Alprazolam 0,5 mg & $1-1 / 2-0-1$ & Si & ¿? & Si \\
\hline
\end{tabular}


Tabla 6 Ficha del paciente para el cuarto SPD

\begin{tabular}{|c|c|c|c|c|c|c|c|c|}
\hline Medicamento & Posología & $\begin{array}{c}\text { Modo de } \\
\text { administración }\end{array}$ & $\begin{array}{c}\text { Vía de } \\
\text { administración }\end{array}$ & $\begin{array}{l}\text { Médico } \\
\text { prescriptor }\end{array}$ & $\begin{array}{l}\text { Fecha inicio } \\
\text { de tratamiento }\end{array}$ & $\begin{array}{l}\text { Fecha prevista } \\
\text { finalización }\end{array}$ & Incidencias & $\begin{array}{l}\text { Blister } \\
\text { Si/No }\end{array}$ \\
\hline $\begin{array}{l}\text { Ezetimiba } \\
10 \mathrm{mg}\end{array}$ & $0-0-1-0$ & $\begin{array}{l}\text { Con o sin } \\
\text { alimentos }\end{array}$ & Oral & MAP & $01 / 15$ & & & Si \\
\hline $\begin{array}{l}\text { Miansenina } \\
30 \mathrm{mg}\end{array}$ & $0-0-0-1$ & $\begin{array}{l}\text { Con o sin } \\
\text { alimentos }\end{array}$ & Oral & Psiquiatra & $04 / 17$ & & & No \\
\hline $\begin{array}{l}\text { Sertralina } \\
50 \mathrm{mg}\end{array}$ & $2-0-0-0$ & $\begin{array}{l}\text { Con o sin } \\
\text { alimentos }\end{array}$ & Oral & Psiquiatra & $21 / 08 / 17$ & & & Sí \\
\hline $\begin{array}{l}\text { Alprazolam } \\
0,5 \mathrm{mg}\end{array}$ & $1 / 2-1 / 2-0-1$ & $\begin{array}{l}\text { Con o sin } \\
\text { alimentos }\end{array}$ & Oral & Psiquiatra & $02 / 16$ & & & Sí \\
\hline $\begin{array}{l}\text { Gabapentina } \\
300 \text { mg }\end{array}$ & $1-0-0-1$ & $\begin{array}{l}\text { Con o sin } \\
\text { alimentos }\end{array}$ & Oral & MAP & $03 / 17$ & & & Sí \\
\hline $\begin{array}{l}\text { Lisinopril } \\
5 \mathrm{mg}\end{array}$ & $1-0-1-0$ & $\begin{array}{l}\text { Con o sin } \\
\text { alimentos }\end{array}$ & Oral & MAP & $05 / 12$ & & & Sí \\
\hline $\begin{array}{l}\text { Nebivolol } \\
5 \mathrm{mg}\end{array}$ & $0-1-0-0$ & $\begin{array}{l}\text { Con o sin } \\
\text { alimentos }\end{array}$ & Oral & MAP & $02 / 16$ & & & Si \\
\hline $\begin{array}{l}\text { Lorazepam } \\
1 \mathrm{mg}\end{array}$ & A demanda & $\begin{array}{l}\text { Con o sin } \\
\text { alimentos }\end{array}$ & Oral & MAP & 2012 & & & No \\
\hline
\end{tabular}

\section{Discusión y conclusiones}

La puesta a disposición de la paciente del servicio de mejora de la adherencia mediante la preparación de SPD ha permitido realizar el cambio en la medicación antidepresiva que le provocaba un problema de ansiedad y la intervención farmacéutica en el proceso de seguimiento condujo a la detección de una intolerancia alimentaria. Además, permitió detectar PRM de conservación de la medicación.

Lo relevante del caso para los autores es que el SPD normalmente se trata como una herramienta a largo plazo, sobre todo en pacientes con dificultades funcionales, que podríamos encuadrar dentro de la categoría de pacientes confundidos tal como describe la guía de adherencia de SEFAC [4]. Tal como se menciona: El paciente confundido suele ser un paciente agobiado por un tratamiento complejo, bien por el número de fármacos, o por la complejidad en la forma de uso de los mismos. Este caso muestra esa minoría, aquellos pacientes que a lo largo de la vida pueden experimentar situaciones de "confusión transitoria", independientes de una categorización de dependencia o de validez de los mismos, que pueden sobrellevarse de forma correcta con el uso de SPD u otras herramientas de personalización de la medicación. Finalmente, no con- viene olvidar que sólo una alianza terapéutica con el paciente, esto es, una relación de confianza y continuada, puede generar los cambios necesarios para una mejora de la adherencia pero que deben existir herramientas adecuadas que los profesionales sanitarios puedan indicar a los pacientes a fin de optimizar los resultados.

Por ello, se proponen las siguientes acciones:

a) la explicitación de subgrupos dentro del grupo de paciente confundido que denominaremos paciente confundido transitorio y paciente confundido dependiente y que recogería la casuística asociada a pacientes no dependientes y dependientes respectivamente.

b) investigar la "confusión transitoria” para determinar, por ejemplo, el número de pacientes afectados por esta situación, el grado de impacto del SPD en estos pacientes y su capacidad para empoderarlos, permitiéndoles superar situaciones que de otro modo podrían desembocar en una exacerbación de la enfermedad.

c) considerar la inclusión del SPD como una herramienta dentro del catálogo de prestaciones del Sistema Nacional de Salud, así como difusión de esta herramienta para atender este tipo de situaciones de "confusión transitoria" entre el resto de los profesionales sanitarios.

\section{Referencias bibliográficas}

1. Decreto 93/2015, de 5 de mayo, del Gobierno de Aragón, por el que se regulan los requisitos y el procedimiento para la dispensación de los Sistemas Personalizados de Dosificación en las oficinas de farmacia en Aragón. Boletín Oficial de Aragón $\mathrm{n}^{\circ}$ 90, de 14/05/2015 [Acceso 12/8/2017] Disponible en http://www.boa.aragon.es/ cgi-bin/EBOA/BRSCGI?CMD=VERDOC\&BASE $=$ BZHT\&PIECE $=$ BOLE\&DO $\mathrm{CR}=13 \mathrm{E}$ SEC $=\mathrm{BUSQUEDA}$ AVANZADAERNG $=10 \& S O R T=-P U B L E S E P A-$ RADOR=CtC ${ }^{*}-\mathrm{C}=$ DECRETO+93/2015

2. Consejo General de Colegios Oficiales de Farmacéuticos [Internet]. Bot PLUS. Base de Datos del Conocimiento Sanitario. 2018. [Acceso 12/2/2018]. Disponible en: http://www.portalfarma. com/inicio/botplus20/Paginas/BotPLUS-2-0.aspx

3. Agencia Española de Medicamentos y Productos Sanitarios (AEMPS) [Internet]. Ficha técnica de paroxetina. [Acceso 12/8/2017]. Disponible en: https://www.aemps.gob.es/cima/pdfs/ es/ft/66218/66218_ft.pdf

4. Prats R. Dispensación, adherencia y uso adecuado del tratamiento: guía práctica para el farmacéutico comunitario. Madrid: Sociedad Española de Farmacia Familiar y Comunitaria (SEFAC); 2017. ISBN: 978-84-6976252. Disponible en: https://www. sefac.org/sites/default/files/2017-11/ Adherencia_0.pdf 